$\mathrm{DE}$

M E D I C I N A

T R O P I C A L

$\mathrm{DE}$

SÃO PAULO

JOURNAL OF THE SÃO PAULO INSTITUTE OF TROPICAL MEDICINE

${ }^{1}$ Ministry of Public Health, National Institute of Health, Department of Medical Sciences, Nonthaburi, Thailand

${ }^{2}$ Mae Sot Hospital, Tak, Thailand

${ }^{3}$ Ministry of Public Health, Medical Sciences Technical Office, Department of Medical Sciences, Nonthaburi, Thailand

Correspondence to: Benjawan Phetsuksiri Ministry of Public Health, Department of Medical Sciences, 88/7 Tiwanond Rd, 11000, Muang, Nonthaburi, Thailand Tel: +66 025801567 ,

Fax: +66 029659700

E-mail: benjapsk@ health.moph.go.th

Received: 1 July 2021

Accepted: 13 October 2021

\section{Putative extensive and pre-extensive drug resistant- tuberculosis associated with unusual genotypes on the Thailand-Myanmar border}

Janisara Rudeeaneksin', Wiphat Klayut', Sopa Srisungngam ${ }^{1}$, Supranee

Bunchoo', Sarawut Toonkomdang ${ }^{2}$, Thanee Wongchai ${ }^{2}$, Nattagarn

Chuenchom $^{2}$, Benjawan Phetsuksiri ${ }^{1,3}$

\section{ABSTRACT}

Extensive drug-resistant tuberculosis (XDR-TB) is highly life threatening and its diagnosis is usually difficult and time-consuming. Here we present the first two cases of XDR and pre-XDR-TB diagnosed in 2018 on the Thailand-Myanmar border, more specifically in Tak province. Rapid detection of XDR-TB was performed by loop-mediated isothermal amplification (LAMP), Xpert MTB/RIF, and line probe assays. Mutation analyses targeting $r p o B, k a t G$, inhA, gyrA and $r r s$ genes showed an association with drug-resistant phenotypes, except for rifampicin resistance. Spoligotyping revealed uncommon Beijing and T2 genotypes and the analysis of $M$. tuberculosis interspersed repetitive unit-variable number tandem repeat (MIRU-VNTR) showed the presence of more polymorphisms. This report highlights the importance of the early detection of drug-resistant tuberculosis by molecular tests followed by phenotyping assays. Based on the up-to-date definition of XDR- and pre-XDR-TB, the susceptibility testing for bedaquiline and linezolid is required and the two reported cases may correspond to putative XDR-TB.

KEYWORDS: Extensively drug-resistant tuberculosis. XDR-TB. Diagnosis. Genotypes. Thailand-Myanmar border.

\section{INTRODUCTION}

Tuberculosis (TB) has remained a global health problem, and extensively drugresistant (XDR)-TB, a dangerous form of TB has emerged as a significant threat worldwide. Until 2020, XDR-TB was defined as resistance to isoniazid (INH), rifampicin (RIF) (multidrug-resistant tuberculosis, MDR), plus any quinolones (FQ), and any second-line injectable drugs (SLID) ${ }^{1}$. In January 2021, the World Health Organization (WHO) endorsed the new definitions of XDR-TB and pre-extensively-TB (pre-XDR-TB). XDR-TB is defined as multidrug-resistant (MDR)-TB or rifampicin-resistant (RR)-TB in conjunction with resistance to any fluoroquinolones and at least one of the additional Group A second-line drugs comprising levofloxacin, moxifloxacin, bedaquiline and linezolid ${ }^{2}$. Pre-XDRTB is MDR-TB/ RR-TB which is also resistant to any fluoroquinolones, while the definition of MDR-TB remains unchanged and is the TB resistant to at least isoniazid (INH) and rifampicin (RIF) ${ }^{2}$.

Thailand is a TB endemic country with an incidence of 150 per 100,000 inhabitants, and over 105,000 cases and about 12,000 deaths were reported in $2020^{3}$, placing Thailand on the list of the top 30 TB-high burden countries. The situation 
is further complicated by drug-resistant TB. In addition, there is an increase in the number of imported cases from Myanmar to the Western border. A high rate of XDR-TB has been reported in Kanchanaburi province located near Myanmar $^{4}$, but there is limited information in its neighbor, Tak province. The high prevalence of drug resistant TB in Thailand and Myanmar has been recognized and the early detection of TB drug resistance through molecular testing is recommended.

Drug-resistant TB was often associated with mutations in specific genes. Notably, rifampicin (RIF) resistance was highly associated with mutations in the $r p o B$ and the hot-spot region is known as the rifampicin resistance determining region (RRDR). Other mutations in the $k a t G$, gyrA, rrs and in the inhA promoter region were frequently detected and accounted for the common resistance to INH ( $k a t G$ and inhA) and kanamycin (rrs), respectively ${ }^{5}$. Among INH resistance mutations, $k a t G$ (Ser315Thr) was the most prevalent, while promoter mutations in the inhA account for a small portion, of about $8-10 \%{ }^{5}$. Hence, mutation analyses can rapidly identify drug-resistant TB strains and provide an insight into the emergence of drug-resistant TB.

To differentiate Mycobacterium tuberculosis (MTB) strains for epidemiological investigations, genotyping has been employed. Spoligotyping is often used and can divide TB strains into several families, mainly the Beijing and different non-Beijing types ${ }^{6,7}$. The Beijing lineage originated in Asia, but has spread and caused outbreaks worldwide. Most Beijing lineages showed the spoligo international number 1 (SIT 1) $)^{5,8}$. In contrast, a study in Latin America found a low prevalence of Beijing genotypes. A prevalent trend of less than 5\% was found, except for Cuba (17.2\%), Peru (16.0\%) and Columbia $(5 \%)^{9}$. Sub-typing of a large group of Beijing strains and clusters of non-Beijing genotypes can be achieved by the mycobacterial interspersed repetitive unit-variable number tandem repeat (MIRU-VNTR) analysis ${ }^{10-12}$. By means of several detection methods followed by genotyping, the first two cases of XDR-TB and pre-XDR-TB on the ThailandMyanmar border in Mae Sot district, located in Tak province, were detected and their molecular characteristics were documented.

\section{CASE REPORT}

In June 2018, samples from 17 MDR-TB and rifampicinresistant (RR)-TB patients were shipped from Tak province to the National Institute of Health in Nonthaburi. Two cases were characterized as XDR-TB (patient 1) and pre-XDR-TB (patient 2), respectively.

\section{Case 1}

Patient 1 was a 56-year-old man who initially sought medical care at the Mae Ramat District Hospital, near the Myanmar border. The screening test by Ziehl Neelsen (ZN) acid-fast bacilli (AFB) microscopy was positive. Sputum was sent for culture at the Mae Sot Hospital that is close to the Mae Ramat District hospital. Repeat ZN smear microscopy showed positive AFB, but the AFB grading was not possible. MTB was firstly detected by loop-mediated isothermal amplification (LAMP) ${ }^{13}$, and Xpert MTB/RIF (Cepheid, Sunnyvale, CA). This patient was diagnosed with RR-TB through GeneXpert testing in May 2017. The direct detection of first-line drug resistance by LPA was not obtained probably be due to a small number of bacilli. Firstly, he was treated for MDR-TB in October 2017 and continued to receive a second-line drug regimen with no cure. Subsequently, Mycobacterial growth was detected in sputum by an automated liquid cultured (BACTEC MGIT 960) following standard protocols. A sputum culture from October 2018 grew MTB using the SD Bioline Ag MPT64 Rapid ${ }^{\circledR}$ (Standard Diagnostics, Korea) for MTB identification, without non-tuberculous mycobacteria (NTM). The culture sample was shipped for the second-line drug testing by line probe assay (LPA) and the secondline drug DST in December 2018. Fluoroquinolones and kanamycin resistance was rapidly identified by LPA using the GenoType ${ }^{\circledR}$ MTBDRplus VER 2.0 (Hain Lifescience, Nehren, Germany) for first-line drugs, resulting in the detection of a rpoB mutation at codon 531 (Ser531Leu), including a kat $G$ mutation at codon 315 (Ser315Thr) and the GenoType ${ }^{\circledR}$ MTBDRs $l$ VER 1.0 assay for second-line drugs which detected a gyrA mutation at codon 94 (Asp94Gly) and a rrs mutation at nucleotide position 1401, respectively. The mutation in the $e m b B$ gene at codon 306 conferring resistance to ethambutol (EMB) was also simultaneously detected by LPA, the GenoType ${ }^{\circledR}$ MTBDRplus VER 2.0 (Table 1).

The phenotypic drug susceptibility testing (DST) was subsequently conducted by the routine proportion method in solid media, and drug concentrations used in DST were summarized in Table 2. DST results confirmed first-line drug resistance to isoniazid, rifampicin, ethambutol and other drugs. Furthermore, the bacilli also showed resistance to second-line drugs: ofloxacin, levofloxacin, kanamycin and others, except for cycloserine (Table 2).

Sequencing analysis using primers described by Poudel et al. ${ }^{14,15}$ identified a $r p o B$ mutation at codon 531 (Ser531Leu) and a katG mutation at codon 315 (Ser315Thr) which were associated with rifampicin and isoniazid resistance, respectively. An additional gyrA 
Table 1 - Detection of DNA mutations associated with isoniazid, rifampicin, ofloxacin and kanamycin resistance in two isolates from XDR-TB and pre-XDR-TB cases using GenoType MTBDRplus and GenoType MTBDRsl.

\begin{tabular}{|c|c|c|c|c|c|c|c|}
\hline \multirow{2}{*}{ Patient $N^{\circ}$} & \multirow{2}{*}{$\begin{array}{l}\text { Collection } \\
\text { period }\end{array}$} & \multicolumn{3}{|c|}{ MTBDRplus } & \multicolumn{3}{|c|}{ MTBDRsI } \\
\hline & & rров & katG & inhA & gyrA & rrs & embB \\
\hline 1 & 2018 & $\begin{array}{c}\text { MUT3 } \\
\text { (codon 531) }\end{array}$ & $\begin{array}{c}\text { MUT1 } \\
\text { (codon 315) }\end{array}$ & WT & $\begin{array}{c}\text { MUT3C } \\
\text { (codon 94) }\end{array}$ & $\begin{array}{c}\text { MUT1 } \\
\text { (codon 1401) }\end{array}$ & $\begin{array}{c}\text { MUT1B } \\
\text { (codon 306) }\end{array}$ \\
\hline 2 & 2018 & WT & $\begin{array}{c}\text { MUT1 } \\
\text { (codon 315) }\end{array}$ & WT & $\begin{array}{l}\text { WT3 missing } \\
\text { (codon 92-97) }\end{array}$ & WT & WT \\
\hline
\end{tabular}

Point mutations in the $r p o B$ gene confers resistance to rifampicin; katG gene and inhA gene promoter mutations confer resistance to isoniazid; the gyrA gene mutation confers resistance to fluoroquinolones; the rrs gene mutation confers resistance to kanamycin; the embB gene mutation confers resistance to ethambutol; WT = wild-type (no resistance); MUT = mutation (resistance) conferred by the gene mutation, with specific mutations indicated as number.

Table 2 - Laboratory detection and molecular characteristics of MTB isolated from a XDR-TB patient (case 1) and a pre-XDR-TB patient (case 2) on theThailand-Myanmar border.

\begin{tabular}{|c|c|c|c|c|}
\hline Laboratory testing & & & $\begin{array}{l}\text { Patient } 1 \\
\text { (XDR-TB) }\end{array}$ & $\begin{array}{c}\text { Patient } 2 \\
\text { (Pre-XDR-TB) }\end{array}$ \\
\hline AFB & & & Positive & Positive \\
\hline LAMP & & & MTB & MTB \\
\hline Xpert MTB/RIF & & & MTB & MTB \\
\hline Culture & & & MTB & MTB \\
\hline \multirow{19}{*}{ Results of DST } & Xpert MTB/RIF & Rifampicin & $\mathrm{R}$ & $\mathrm{S}$ \\
\hline & \multirow{2}{*}{$\begin{array}{c}\text { LPA } \\
1^{\text {st }} \text { line (MTBDRplus) }\end{array}$} & Rifampicin $(r p o B)$ & $\mathrm{R}$ & $\mathrm{S}$ \\
\hline & & Isoniazid (katG/inhA) & $\mathrm{R}$ & $\mathrm{R}$ \\
\hline & \multirow{2}{*}{$\begin{array}{c}\text { LPA } \\
2^{\text {nd }} \text { line (MTBDRs/) }\end{array}$} & $\begin{array}{l}\text { Fluoroquinolones } \\
\qquad(\text { gyrA) }\end{array}$ & $\mathrm{R}$ & $\mathrm{R}$ \\
\hline & & $\mathrm{AG} / \mathrm{CP}(r r s)$ & $\mathrm{R}$ & $\mathrm{S}$ \\
\hline & \multirow{4}{*}{$\begin{array}{c}\text { Solid DST } \\
1^{\text {st }} \text { line }(\mu \mathrm{g} / \mathrm{mL})\end{array}$} & Rifampicin (40.0) & $\mathrm{R}$ & $\mathrm{R}$ \\
\hline & & Isoniazid $(0.2 / 1.0)$ & $\mathrm{R}$ & $\mathrm{R}$ \\
\hline & & Streptomycin (4.0) & $\mathrm{R}$ & $S$ \\
\hline & & Ethambutol (2.0) & $\mathrm{R}$ & $\mathrm{S}$ \\
\hline & \multirow{6}{*}{$\begin{array}{c}\text { Solid DST } \\
2^{\text {nd }} \text { line }(\mu \mathrm{g} / \mathrm{mL})\end{array}$} & Ofloxacin (2.0) & $\mathrm{R}$ & $\mathrm{R}$ \\
\hline & & Kanamycin (30.0) & $\mathrm{R}$ & S \\
\hline & & Levofloxacin (2.0) & $\mathrm{R}$ & $\mathrm{R}$ \\
\hline & & Cycloserine (40.0) & $\mathrm{S}$ & $S$ \\
\hline & & PAS (1.0) & $\mathrm{R}$ & $S$ \\
\hline & & Capreomycin (40.0) & $\mathrm{R}$ & $\mathrm{S}$ \\
\hline & \multirow{2}{*}{$\begin{array}{l}\text { DNA sequencing } \\
1^{\text {st line }}\end{array}$} & rров & $\begin{array}{l}\text { Ser531Leu } \\
\text { (TCG } \rightarrow \text { TTG) }\end{array}$ & Wild-type \\
\hline & & katG & $\begin{array}{c}\text { Ser315Thr } \\
(\mathrm{AGC} \rightarrow \mathrm{ACC})\end{array}$ & $\begin{array}{c}\text { Ser315Thr } \\
(\mathrm{AGC} \rightarrow \mathrm{ACC})\end{array}$ \\
\hline & \multirow{2}{*}{$\begin{array}{l}\text { DNA sequencing } \\
2^{\text {nd }} \text { line }\end{array}$} & gyrA & $\begin{array}{c}\text { Asp94Gly } \\
\text { (GAC } \rightarrow \text { GGC) }\end{array}$ & $\begin{array}{c}\text { Asp94Tyr } \\
(\mathrm{GAC} \rightarrow \text { TAC) }\end{array}$ \\
\hline & & rrs & $\mathrm{A} 1401 \mathrm{G}$ & Wild-type \\
\hline \multirow{3}{*}{ Genotyping } & \multirow{2}{*}{ Spoligotyping } & Spoligotype & Beijing & $\mathrm{T} 2$ \\
\hline & & SIT no. & 941 & 52 \\
\hline & \multicolumn{2}{|l|}{ MIRU-VNTR typing* } & 4423335434553823 & 3422333124135832 \\
\hline \multicolumn{5}{|c|}{$\begin{array}{l}\text { *Genetic markers located in } 16 \text { loci, in order: VN424 (424), ETR-C (577), MIRU04 (580), MIRU40 (802), MIRU10 (960), MIRU16 } \\
\text { (1644), VN1955 (1955), QUB11b (2163b), ETR-A (2165), VN2401 (2401), MIRU26 (2996), MIRU31 (3192), VN3690 (3690), } \\
\text { QUB26 (VN4052), VN4156 (4156) and MIRU39 (4348); AFB = acid-fast bacilli; LAMP = loop-mediated isothermal amplification; } \\
\text { MTB = Mycobacterium tuberculosis; DST = drug susceptibility testing; LPA = line probe assay; AG/CP = aminoglycosides/cyclic } \\
\text { peptides; PAS = para-aminosalicylic acid; MIRU-VNTR = mycobacterial interspersed repetitive unit-variable number tandem repeat; } \\
\text { R = resistant; S = susceptible. }\end{array}$} \\
\hline
\end{tabular}


mutation at codon 94 (Asp94Gly) conferring resistance to fluoroquinolones and a rrs mutation at nucleotide position 1401 conferring resistance to kanamycin were confirmed. The DNA mutations detected by sequencing and LPA were consistent (Tables 1 and 2). Based on DST and LPA results that resulted in gyrA MUT3C and rrs MUT1 types, the patient was finally diagnosed as having a XDR-TB in 2018. The long and complicated history of the disease was noteworthy. Finally, he was treated at that time to cure XDR-TB according to the WHO recommended standard regimen, while the contactants investigation did not reveal transmission of XDR-TB.

\section{Case 2}

This male patient had no history information since he was an immigrant. RR-TB was identified only by solid DST. No mutation in the $r p o B$ gene was detected by Xpert MTB/RIF and LPA including the targeted sequencing. The phenotypic profile showed resistance to rifampicin, isoniazid in addition to resistance to ofloxacin and levofloxacin, but not to kanamycin. LPA detected a $k a t G$ mutation at codon 315 (Ser315Thr) and a gyrA mutation somewhere between codons 92-97. Sequencing confirmed the kat $G$ mutation at codon 315 (Ser315Thr) and the gyrA at codon 94 (Asp94Tyr), respectively. DNA mutations involved in first and second-line drug resistance, as detected by LPA and sequencing, were summarized in Tables 1 and 2. Based on DST and LPA results with gyrA wild-type 3 missing types, the patient was finally diagnosed as having a pre-XDR-TB. The patient was lost during the follow-up.

Overall, mutations in the $k a t G, r p o B$, gyrA and rrs genes were associated with drug-resistant phenotypes, except for rifampicin resistance in patient 2. Consistency of results was identified between molecular testing and sequencing. By the genotypic polymorphism analysis, spoligotyping revealed the Beijing genotype with spoligo international type number (SIT no.) 941 in case 1, and T2 with SIT no. 52 in case 2 . The spoligotypes patterns are shown in Figure 1. MIRU-VNTR typing which was performed based on the standard 15-locus MIRU-VNTR described by Supply et al. ${ }^{10}$ as well as the MIRU39 (4348) locus analysis with high allelic diversity ${ }^{5}$ showed different MIRU-VNTR patterns (Table 2).

\section{DISCUSSION}

Different molecular methods were used in an attempt to timely identify the XDR and pre-XDR TB. The method included LAMP, Xpert MTB/RIF, LPA (GenoType ${ }^{\circledR}$ MTBDRplus in addition to the GenoType ${ }^{\circledR} \mathrm{MTBDR} s l$ ) and sequencing. It should be noted that even using molecular tests, the detection for definitive diagnosis of pre- and XDR-TB was time consuming. Generally, phenotypic testing is used to determine the susceptibility in all confirmed new TB cases. Since the vast majority of drug-resistant MTB is caused by point mutations and possibly by insertion or deletions, genotypic methods have been applied to screen drug-resistant TB without the constraints of TB culture and the related bio-safety concerns. Therefore, molecular assays represent a valuable option to accelerate the detection of drug resistance to TB.

For a rapid detection, firstly we detected the common mutations in the major hotspots of kat $G$, inh $A$ and $r p o B$ genes. The most common mutation in the $k a t G$ gene (Ser315Thr) is associated with resistance to INH and was identified in both isolates, while the most common rpoB mutation at Ser531Leu (Ser450Leu) is associated with resistance to RIF and was identified only in the XDR-TB case. On the other hand, we found that RR was missed in the detection by molecular tests in the pre-XDR-TB. The subsequent phenotypic testing verified the accuracy of previous results, and proved its value especially in patients suspected of having MDR- or XDR-TB. Actually, the sensitivity of genotypic tests for the detection of RR, a surrogate for the MDR-TB marker, is $95 \%{ }^{16,17}$ based on the evidence that most RIF resistant strains contain $r p o B$ mutations located in RRDR. The lack of RR detection in the pre-XDR-TB may be due to an uncommon mechanism of RR in this isolate that could be mediated by rare specific mutations in the $r p o B$ gene and may be located outside the $\mathrm{RRDR}^{18}$. Other factors such as critical concentrations of drugs used, and random errors could also play a role. Furthermore, mutations indicating resistance to fluoroquinolones or injectable aminoglycosides were also found in gyrA and $r r s$ genes, respectively. The two isolates contained the most common gyrA (Asp94Gyr and rrs (A1401G) mutations. To elucidate the different mechanisms of RR and possibly other mutations, whole genome sequencing should be applied.

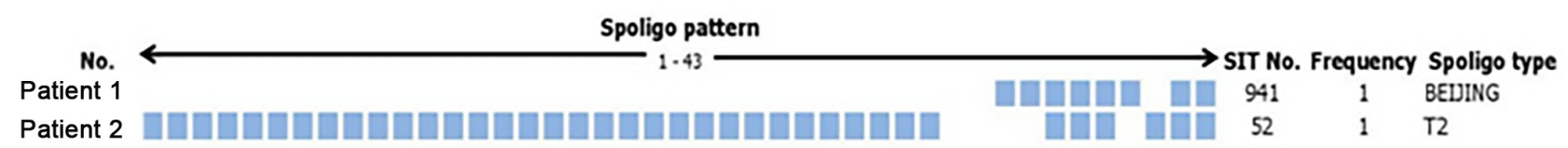

Figure 1 - Spoligotyping results of the XDR-TB patient (case 1), and the pre-XDR-TB patient (case 2). 
For genotyping, spoligotyping and the limited 16-locus MIRU-VNTR analysis were applicable and were able to identify the uncommon Beijing and $\mathrm{T} 2$ genotypes with specific MIRU-VNTR patterns. In general, the characteristic of the Beijing type is a higher rate of mutations, which facilitate the emergence of drug resistance ${ }^{19}$. The predominant Beijing lineage often shows the SIT 1, usually forming a large cluster of isolates and the MIRU-VNTR typing was used for subtypes discrimination ${ }^{4}$. In this report, genetic polymorphism revealed Beijing lineage with the rare SIT at 941 position in the XDR-TB, while the preXDR strain was a T2 lineage, a genotype found frequently in Africa, but not in $\mathrm{Asia}^{20}$. MIRU-VNTR typing further demonstrated the genetic difference of the two isolates. The MIRU39 locus analysis was added since it has been reported that ETR-C of the standard 15-locus MIRUVNTR has a low discriminatory power among Beijing lineage samples ${ }^{12}$. This locus exhibited polymorphisms in our analysis (Table 2). The baseline information of the genotypes reported here would be valuable for tracking the transmission of highly drug-resistant TB bacilli in the future. The migrant patient who was lost to followup was at risk for TB control. To our knowledge, no new XDR-TB cases have been identified up to the writing of this report. It should be noted that drug resistance pattern of MDR-/RR-TB including FQ and the group A drugs (levofloxacin/ molxifloxacin, linezolid and bedaquiline are currently applied for the identification of XDR-TB and pre-XDR-TB. According to the WHO new definitions of XDR-TB and pre-XDR-TB, these two cases of highly drugresistant TB might be XDR or pre-XDR-TB, as both were MDR-TB with additional resistance to the fluoroquinolones olfloxacin and levofloxacin. The phenotypic drug susceptibility testing (DST) for bedaquiline and linezolid is in need and hence poses some challenges. This report can at least stimulate the local capacity building for DSTs of these new drugs and other repurposed medicines in the context of TB.

This document reports the first two cases of possible XDR-TB and pre-XDR-TB with unusual genotypes on the Thailand-Myanmar border, Tak province. The usefulness of genotyping as well as the rapid detection of TB and the drug resistance of bacilli revealed by genotying methods were highlighted in the present report. Although culturebased DST is considered the gold standard, phenotypic detections are not always conclusive to detect resistance, but it is required in suspicious cases in which the molecular detection has failed. The disagreements between genotypic and phenotypic drug-resistant testings should be further explored.

\section{ACKNOWLEDGMENTS}

This study was funded by the Health System Research Institute (HSRI), grant No. 60-086. The cooperation of the Mae Sot Hospital and the support of the Bureau of Tuberculosis are acknowledged.

\section{CONFLICT OF INTERESTS}

None to declare.

\section{REFERENCES}

1. World Health Organization. Definitions and reporting framework for tuberculosis - 2013 revision: updated December 2014 and January 2020. Geneva: WHO; 2013. [cited 2021 Nov 5]. Available from: https://apps.who.int/iris/handle/10665/79199

2. World Health Organization. WHO announces updates definitions of extensively drug resistant tuberculosis. Geneva: WHO; 2021. [cited 2021 Nov 5]. Available from: https://www.who.int/ news/item/27-01-2021-who-announces-updated-definitionsof-extensively-drug-resistant-tuberculosis

3. World Health Organization. Global tuberculosis report 2020. Geneva: WHO; 2020. [cited 2021 Nov 5]. Available from: https://www.who.int/publications/i/item/9789240013131

4. Disratthakit A, Meada S, Prammananan T, Thaipisuttikul I, Doi N, Chaiprasert A. Genotypic diversity of multidrug-, quinoloneand extensively drug-resistant Mycobacterium tuberculosis isolates in Thailand. Infect Genet Evol. 2015;32:432-9.

5. San LL, Aye KS, Oo NA, Shwe MM, Fukushima Y, Gordon $\mathrm{SV}$, et al. Insight into multidrug-resistant Beijing genotype Mycobacterium tuberculosis isolates in Myanmar. Int J Infect Dis. 2018;76:109-19.

6. Kamerbeek J, Schouls L, Kolk A, van Agterveld M, van Soolingen D, Kuijper S, et al. Simultaneous detection and strain differentiation of Mycobacterium tuberculosis for diagnosis and epidemiology. J Clin Microbiol. 1997;35:907-14.

7. Goguet de la Salmonière YO, Li HM, Torrea G, Bunschoten A, van Embden J, Gicquel B. Evaluation of spoligotyping in a study of the transmission of Mycobacterium tuberculosis. J Clin Microbiol. 1997;35:2210-4.

8. Rudeeaneksin J, Phetsuksiri B, Nakajima C, Bunchoo S, Suthum K, Tipkrua N, et al. Drug-resistant Mycobacterium tuberculosis and its genotypes isolated from an outbreak in western Thailand. Trans R Soc Trop Med Hyg. 2021;115:886-95.

9. Cerezo-Cortés MI, Rodríguez-Castillo JG, Hernández-Pando R, Murcia MI. Circulation of M. tuberculosis Beijing genotype in Latin America and the Caribbean. Pathog Glob Health. 2019;113:336-51.

10. Supply P, Allix C, Lesjean S, Cardoso-Oelemann M, RüschGerdes S, Willery E, et al. Proposal for standardization of 
optimized mycobacterial interspersed repetitive unit-variablenumber tandem repeat typing of Mycobacterium tuberculosis. J Clin Microbiol. 2006;44:4498-510.

11. Li Y, Cao X, Li S, Wang H, Wei J, Liu P, et al. Characterization of Mycobacterium tuberculosis isolates from Hebei, China: genotypes and drug susceptibility phenotypes. BMC Infect Dis. 2016;16:107.

12. Wang J, Liu Y, Zhang CL, Ji BY, Zhang LZ, Shao YZ, et al. Genotypes and characteristics of clustering and drug susceptibility of Mycobacterium tuberculosis isolates collected in Heilongjiang Province, China. J Clin Microbiol. 2011;49:1354-62

13. Phetsuksiri B, Rudeeaneksin J, Srisungngam S, Bunchoo S, Klayut W, Sangkitporn S, et al. Loop-mediated isothermal amplification for rapid identification of Mycobacterium tuberculosis in comparison with immunochromatographic SD Bioline MPT64 Rapid ${ }^{\circledR}$ in a high burden setting. Jpn J Infect Dis. 2019;72:112-4.

14. Poudel A, Nakajima C, Fukushima Y, Suzuki H, Pandey BD, Maharjan B, et al. Molecular characterization of multidrugresistant Mycobacterium tuberculosis isolated in Nepal. Antimicrob Agents Chemother. 2012;56:2831-6.

15. Poudel A, Maharjan B, Nakajima C, Fukushima Y, Pandey BD, Beneke A, et al. Characterization of extensively drug-resistant
Mycobacterium tuberculosis in Nepal. Tuberculosis (Edinb). 2013;93:84-8.

16. Ritter C, Lucke K, Sirgel FA, Warren RW, van Helden PD, Böttger EC, et al. Evaluation of the aid TB resistance line probe assay for rapid detection of genetic alterations associated with drug resistance in Mycobacterium tuberculosis strains. J Clin Microbiol. 2014;52:940-6.

17. Denkinger CM, Schumacher SG, Boehme CC, Dendukuri N, Pai M, Steingart KR, et al. Xpert MTB/RIF assay for the diagnosis of extrapulmonary tuberculosis: a systematic review and metaanalysis. Eur Respir J. 2014;44:435-46.

18. Ma P, Luo T, Ge L, Chen Z, Wang X, Zhao R, et al. Compensatory effects of $M$. tuberculosis rpoB mutations outside the rifampicin resistance-determining region. Emerg Microbes Infect. 2021;10:743-52.

19. Merker M, Blin C, Mona S, Duforet-Frebourg N, Lecher S, Willery E, et al. Evolutionary history and global spread of the Mycobacterium tuberculosis Beijing lineage. Nat Genet. 2015;47:242-9.

20. Lukoye D, Katabazi FA, Musisi K, Kateete DP, Asiimwe BB, Okee M, et al. The T2 Mycobacterium tuberculosis genotype, predominant in Kampala, Urganda, shows negative correlation with antituberculosis drug resistance. Antimicrob Agents Chemother. 2014;58:3853-9. 